\section{The regional specificity of rapid actions of cocaine}

\author{
Brandon J. Aragona
}

In their recent Perspective article, Roy Wise and Eugene Kiyatkin conclude that, "the primary, unconditioned rewarding effects of cocaine in naive animals involves the blockade of dopamine reuptake rather than stimulation of dopamine release" (Differentiating the rapid actions of cocaine. Nature Rev. Neurosci. 12, 479-484 (2011)) $)^{1}$. However, recent voltammetry studies that have measured realtime changes in dopamine concentration at the instant that cocaine enters the brain have reached different conclusions ${ }^{2,3,4,5}$. These studies show that intravenous cocaine delivery inhibits dopamine uptake in less than 5 seconds following drug delivery ${ }^{4,5}$ and increases the frequency of phasic dopamine release events within the nucleus accumbens (NAc) shell to a greater extent than in the NAc core ${ }^{2,3,6,7}$. This shell-specific increase in dopamine release begins approximately 10 seconds after cocaine delivery and lasts for approximately 2 minutes ${ }^{2}$. Unconditioned cocaine-evoked dopamine release is therefore both temporally and regionally specific and is important because it mediates the primary rewarding effects of cocaine ${ }^{3,8}$.

Conversely, cocaine-predictive cues cause a very brief and rapid surge in dopamine concentration within the NAc core, but not the NAc shell ${ }^{3,9-11}$ (FIG. 1A). Thus, the unconditioned and conditioned dopamine release that are evoked by cocaine occur in anatomically and functionally distinct mesolimbic dopamine projection would not apply in this case. systems ${ }^{2,3,12-14}$. It is therefore problematic that Wise and Kiyatkin refer to 'dopamine reward' or 'the dopamine system'. For example, they predict that interoceptive cues that are associated with intravenous drug delivery will evoke conditioned dopamine release in rats with cocaine self-administration experience because these cues are extremely reliable predictors of cocaine's effects in the brain and, "immediate activation of the dopamine system occurs when the reward is reliably predicted by environmental stimuli ... [because food reward studies show that] ... reward predictors can activate the dopamine system before the receipt of the reward itself." However, dopamine release that is evoked by food reward and food-predictive cues occurs within the same brain region, the NAc core ${ }^{15,16}$, whereas cocaine and cocainepredictive cues increase dopamine release in different brain regions (the NAc shell and core, respectively $)^{2,3,10,11}$. Therefore, the conventional 'transfer' of phasic dopamine release from the primary reward to the reward-predictive cue

Thus, one must take into account the specific dopamine system that is involved when generating predictions about unconditioned dopamine release evoked by a primary reward or about the development of conditioned dopamine release by reward-predictive cues. Moreover, a recent voltammetry study showed that phasic dopamine release within the NAc core transfers from
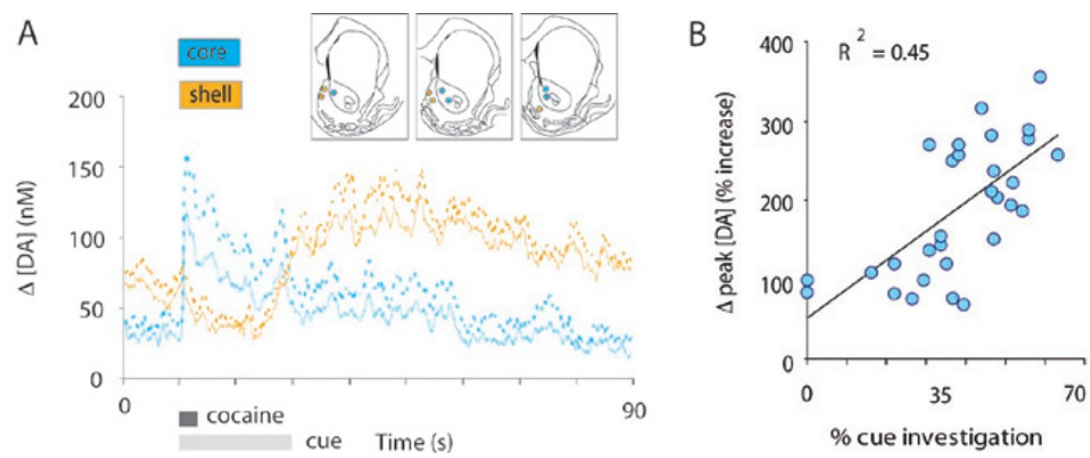

Figure 1 | Real-time dopamine transmission during a Pavlovian cue-cocaine association. A|Sub-second changes in dopamine (DA) concentration within the nucleus accumbens (NAc) core (shown in blue) and NAc shell (shown in orange) (inset shows measurement locations; solid lines show the mean, dashed lines show the mean + standard error). Following 1 day of Pavlovian training, presentation of a cocaine-predictive cue evokes increases in DA concentration within the NAc core but decreases in DA concentration within the NAc shell. B | Cue-evoked increases in DA concentration within the NAc core are correlated with the extent of Pavlovian approach behaviour (cue investigation) that is directed towards the cocaine-predictive cue. Figure is reproduced, with permission, from REF 3 ๑ (2009) Wiley-Blackwell. the primary reward to the reward-predictive cue only when the reward-predictive cue has substantial incentive value (that is, when it generates Pavlovian approach $)^{16}$. A similar relationship between dopamine release and Pavlovian approach has been shown for cocaine-predictive cues $^{3}$ (FIG. 1B). As such, the incentive value of the stimulus should also be considered when predicting how dopamine release may be related to the interoceptive cues that are related to intravenous cocaine delivery, as these cues cannot be associated with behavioural approach and may therefore have low incentive value.

Brandon J. Aragona is at the Department of Psychology and Neuroscience Program, University of Michigan, Ann Arbor, Michigan 48109-1043, USA. e-mail:aragona@umich.edu doi:10.1038/nrn3043-c1 Published online 5 October 2011

Wise, R. A. \& Kiyatkin, E. A. Differentiating the rapid actions of cocaine. Nature Rev. Neurosci. 12 , 479-484 (2011)

2 Aragona, B. J., Cleaveland, N. A., Stuber, G. D., Day, J. J., Carelli R. M. \& Wightman, R. M. Preferential enhancement of dopamine transmission within the nucleus accumbens shell by cocaine is attributable to a direct increase in phasic dopamine release events. J. Neurosci. 28, 8821-8831 (2008).

3. Aragona, B. J., Day, J. J., Roitman, J. J., Cleaveland, N. A., Wightman, R. M. \& Carelli, R. M. Regional specificity in the real-time development of phasic dopamine transmission patterns during acquisition of a cue-cocaine association in rats. Eur. J. Neurosci. 30, 1889-1899 (2009).

4. Espana, R. A., Roberts, D. C. \& Jones, S. R. Shortacting cocaine and long-acting GBR-12909 both elicit rapid dopamine uptake inhibition following intravenous delivery. Neuroscience 155, 250-257 (2008).

5. Mateo, Y. Budygin, E. A., Morgan, D., Roberts, D. C. $\bar{d}$ Jones, S. R. Fast onset of dopamine uptake inhibition by intravenous cocaine. Eur. J. Neurosci. 20 2838-2842 (2004).

6. Cheer, J. F. et al. Phasic dopamine release evoked by abused substances requires cannabinoid receptor activation. J. Neurosci. 27, 791-795 (2007).

Sombers, L. A., Beyene, M., Carelli, R. M. \& Wightman, R. M. Synaptic overflow of dopamine in the nucleus accumbens arises from neuronal activity in the ventral tegmental area. J. Neurosci. 29, 1735-1742 (2009).

8. Di Chiara, G. \& Bassareo, V. Reward system and addiction: what dopamine does and doesn't do. Curr. Opin. Pharmacol. 7, 69-76 (2007).

9. Ito, R., Dalley, J. W., Howes, S. R., Robbins, T. W. \& Everitt, B. J. Dissociation in conditioned dopamine release in the nucleus accumbens core and shell in response to cocaine cues and during cocaine-seeking behavior in rats. J. Neurosci. 20, 7489-7495 (2000).

10. Phillips, P. E., Stuber, G. D., Heien, M. L., Wightman, R. M. \& Carelli, R. M. Subsecond dopamine release promotes cocaine seeking. Nature 422, 614-618 (2003).

11. Stuber, G. D., Roitman, M. F., Phillips, P. E., Carelli, R. M. \& Wightman, R. M. Rapid dopamine signaling in the nucleus accumbens during contingent and noncontingent cocaine administration. Neuropsychopharmacology 30, 853-863 (2005).

12. Ikemoto, S. Dopamine reward circuitry: two projection systems from the ventral midbrain to the nucleus accumbens-olfactory tubercle complex. Brain Res. Rev. 56, 27-78 (2007).

13. Lammel, S., Ion, D. I., Roeper, J. \& Malenka, R. C. Projection-specific modulation of dopamine neuron synapses by aversive and rewarding stimuli. Neuron 70, 855-862 (2011)

14. Liu, Z. H., Shin, R. \& Ikemoto, S. Dual role of medial A10 dopamine neurons in affective encoding. Neuropsychopharmacology 33 , 3010-3020 (2008).

15. Day, J. J., Roitman, M. F., Wightman, R. M. \& Carelli, R. M. Associative learning mediates dynamic shifts in dopamine signaling in the nucleus accumbens. Nature Neurosci. 10, 1020-1028 (2007).

16. Flagel, S. B. et al. A selective role for dopamine in stimulus-reward learning. Nature 469, 53-57 (2011). 